\title{
Further Research and Applications in the Field of Ozone Therapy: Much more than a "Therapeutic Unconventional Hypothesis"
}

\author{
${ }^{1}$ Lamberto Re MD, ${ }^{1}$ Giuseppe Malcangi MD, ${ }^{2}$ Cristina Gagliardi PhD and \\ 2Oriano Mercante MD. \\ ${ }^{1}$ Medinat SAS, Pharmacology \& Ozone Therapy Department, Camerano, Ancona, Italy, \\ 2Scientific Direction, INRCA-IRCS (National Institute of Health and Science on Aging), Ancona, Italy.
}

doi: 10.7203/jo3t.1.1.2015.12165

Received: October, 2015

Published: December 2015

\section{ABSTRACT}

Ozone therapy is widely used in many countries since many years. Recently, the increasing widespread of this complementary therapy has been accomplished by an increased number of basic and clinic papers published on international journals. Many of the basic mechanisms of the ozone action are now well outlined. In addition, the modulation of interleukins and of some biochemical pathways related to inflammation and pain, indicates the rationale for its use in many pathological conditions related to pain, inflammation and age disorders. We will discuss on the mode of action of ozone that, with a hormetic mechanism, appear to be more similar to xenobiotic stress model than a pure pharmacological action. Indeed, our recent work in vivo on patients treated with major autohemo therapy (MAH) demonstrated the increase of Nrf2 level $(\mathrm{P}<0.01)$ in peripheral blood mononuclear cells (PBMC) immediately after exposition of blood to ozone and prior reinfusion. The activities of superoxide dismutase and catalase were increased $(\mathrm{P}<0.05)$ following the cycle of MAH's. These data demonstrate by the first time in vivo the activation of the Nrf2 pathway by a low dose of ozone and the activation of the feedback mechanism that induces the synthesis of proteins which finally favours the cell survival. Following the above research on Nrf2, it is now in progress a new scientific approach in the aim to study the transcriptional profile by qRT-PCR of transcripts in both Nrf2- and NFkB-dependent genes to further define the profile of the positive effect induced by low ozone doses in many ailments in patients in vivo.

\section{REVIEW}

In light of the growing interest about ozone therapy, we were asked to update - both from a scientific and clinical point of view - the latest applications in the field of Human Medicine with this minireview.

In many conferences in which we are invited to participate as lecturers, we often observe how many doctors and health professionals are increasingly drawn to listening the most recent acquisitions in the field of Ozone Therapy. This testifies that the time is ripe for wider recognition of this therapeutic technique, as indeed was the case recently in the

\section{Authors Information}

\section{E-mail address: lambertore@lambertore.com}

United Arab Emirates where, as a result of a conference organized in April of the last year in Abu Dhabi (http://www.lotusholistic.ae/education/ event/event-details/?e=992\&t=1), the authorities of HAAD (Health Authority Abu Dhabi) officially introduced the specialization of GP Ozone Therapist in the database of the allowed nonconventional or alternative therapies.

To our opinion, it is time that public opinion and health care professionals widen their horizons on ozone therapy. Indeed, this therapeutic approach must be considered not only a resource devoted to the resolution of back pain or herniated disc, but also as a systemic treatment useful in numerous pathological conditions or simply as a therapeutic prophylactic resource to prevent the damages of aging and to improve many functions of our bodies.

Before considering the topics of the latest scientific discoveries, allow us to introduce a new 
terminology regarding the pharmacological mechanism of action underlying the treatment with ozone, which can't be considered - according to classical schemes of pharmacology currently spread in the faculties of medicine - as a simple interaction between the molecule (drug) and the receptor (cellular membrane protein), but rather as "Hormetic Stress".

Our scientific conviction that a molecule like ozone, i.e. a strong oxidant, could induce benefits in various ailments, when used at low doses was believed to be an unconventional theory. We are now very happy to note how this concept could finally gain a certain scientific credibility. Indeed, in a recent paper the Nobelist Dr. James Watson proposed an unconventional view on oxidative stress and diabetes. The conventional view is that oxidative stress causes insulin resistance.

In March of this year, Dr. James Watson, who codiscovered the double helix structure of DNA, proposed an unconventional view on the cause of diabetes. [1]. "The fundamental cause, I suggest, is a lack of biological oxidants, not an excess," he says. "Physical exercise prompts the body to make large numbers of oxidants - molecules called reactive oxygen species, or ROS," he continues, and that's why exercise is beneficial to our bodies. This hypothesis certainly needs to be tested. It could be that it's the balance that matters, and that disease results due to an imbalance on either side; i.e. both oxidative stress or oxidative deficiency (such as hypoxia), could lead to insulin resistance and other diseases. The idea that similar to brief exercise, the conditioning effects induced by small ozone doses, could be helpful for many of the biological functions of all the cells that burn oxygen to produce energy seems to be worthy of new and strong scientific studies dedicated to the matter.

Like other Xenobiotics, agents not recognized in the metabolism of the body (from the Greek Xenos $=$ Foreign and Bios = Life), such as heat, mechanical trauma, ionizing radiation or the same foods that we eat daily, even "ozone" molecule is able to influence the cellular functions. Indeed, following the stress, cells promote protection mechanisms that defend from the specific damage induced by the same stressing agent. The term xenobiotic has been introduced only recently [2], and the details are still lacking of adequate scientific support regarding the involved mechanisms. As is obvious, this is reflected negatively in regards to ozone therapy too, and makes understandable, but not reasonable, the lack of attention of health authorities devoted to the control of human health.

The studies to date are mostly directed to the characterization of the Mode of Action of the Stress Response (MOA), defined as the study of the individual metabolic pathways activated at the cellular level by the various Xenobiotics. Ozone, like other agents, and unlike the common drugs that act on a specific receptor, induces small stress to the whole cell when used at low doses. This, in turn, triggers a series of intracellular metabolic processes and promotes a myriad of intracellular activities. As a consequence of these reactions, the defense mechanisms of the cell are alarmed and enhanced to improve functionality, explaining in part the surprising therapeutic actions of this gas. A recent study published in the prestigious Toxicological Sciences [3] has fully explained the biochemical mechanism with its intracellular mediators (Transducers, Sensors and Transfer Factor), where for each type of xenobiotic is possible to monitor the intracellular metabolic pathways (Tab. 1).

There is no doubt that this complexity makes us understand why it is not easy set up clinical trials demonstrating definitively the therapeutic power of these agents. On the other hand, any drug interaction, due to its direct bind with a receptor and consequent biological function activated, is easily measurable and statistically standardized. In short, the stimulus of Oxidative Stress, in the case of ozone, is able to activate Nrf2 protein $[4 ; 5]$ which, moving into the nucleus, starts production from part of Target Genes of proteins that promote cell functions (Fig. 1), strengthening the defenses, and optimizing the underlying specific function.

We can now start to understand why after the administration of low ozone doses we can observe positive effects, like aesthetics, if the conditioned cell is part of the skin tissue or a modulating effect on inflammation if the cell is in the context of neuromuscular or bone tissues (Fig. 2).

It is time that he various ozone therapy societies, in Italy and internationally, start to increase their scientific weight, being now ozone therapy a common treatment in most of the continents and administered to millions of patients, with virtually no or few side effects (mostly of instrumental or iatrogenic origin). The lack of dedicated university courses and precise legislation at government level, make the sector unstable at present.

Ozone therapy, like other similar holistic approaches, could be included in the newly branch 
Table 1. Metabolic Pathways Messengers

\begin{tabular}{llll}
\hline Pathway & TF & Sensor & Major transducers \\
\hline Oxidative stress & Nrf2 & Keap1 & MAPK, ERK, p38, PKC \\
Heat shock response & HSF-1 & Hsp90 & CaMK2, CK2 \\
DNA damage & p53 & MDM2 & ATM, JNK, Chk1, Chk2 \\
Hypoxia & HIF-1 & VHL & p38, PI3K \\
ER stress & XBP-1, ATF6, ATF4 & BiP & IRE1a, s2P \\
Metal stress & MTF-1 & None & PKC, CKII, TKs \\
Inflammation & NF-KB & IkB & IKK \\
Osmotic stress & NFAT5 & None & p38,ATM,PKA \\
\hline
\end{tabular}

Transducers, Sensors and Transfer Factors of the metabolic pathways involved in stress, from Simmons et al, TOXICOLOGICAL SCIENCES 111 (2), 202-225 (2009)

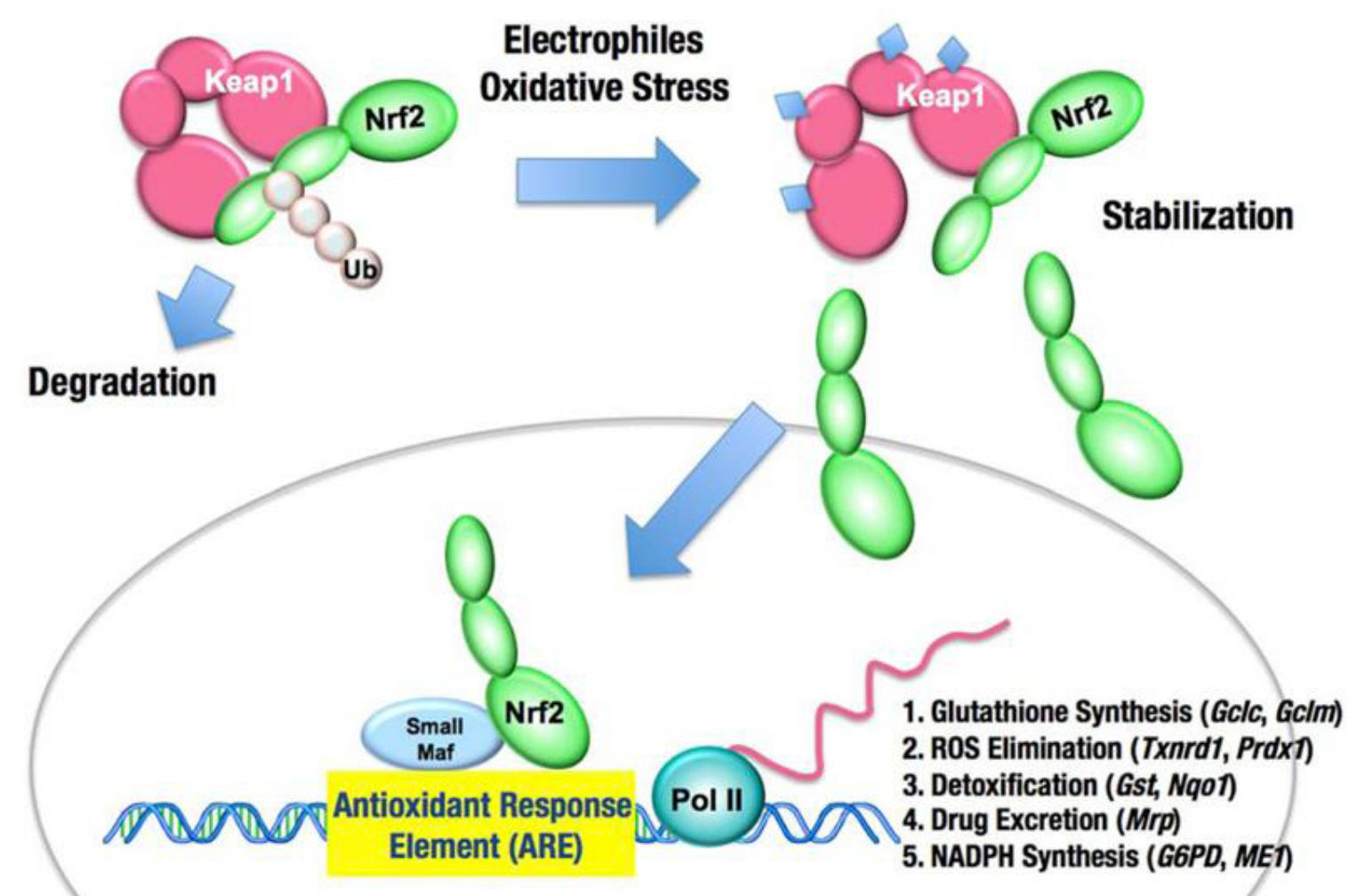

Fig 1. Schematic Pathways of Nrf2 and Antioxidant Response Elements activation 


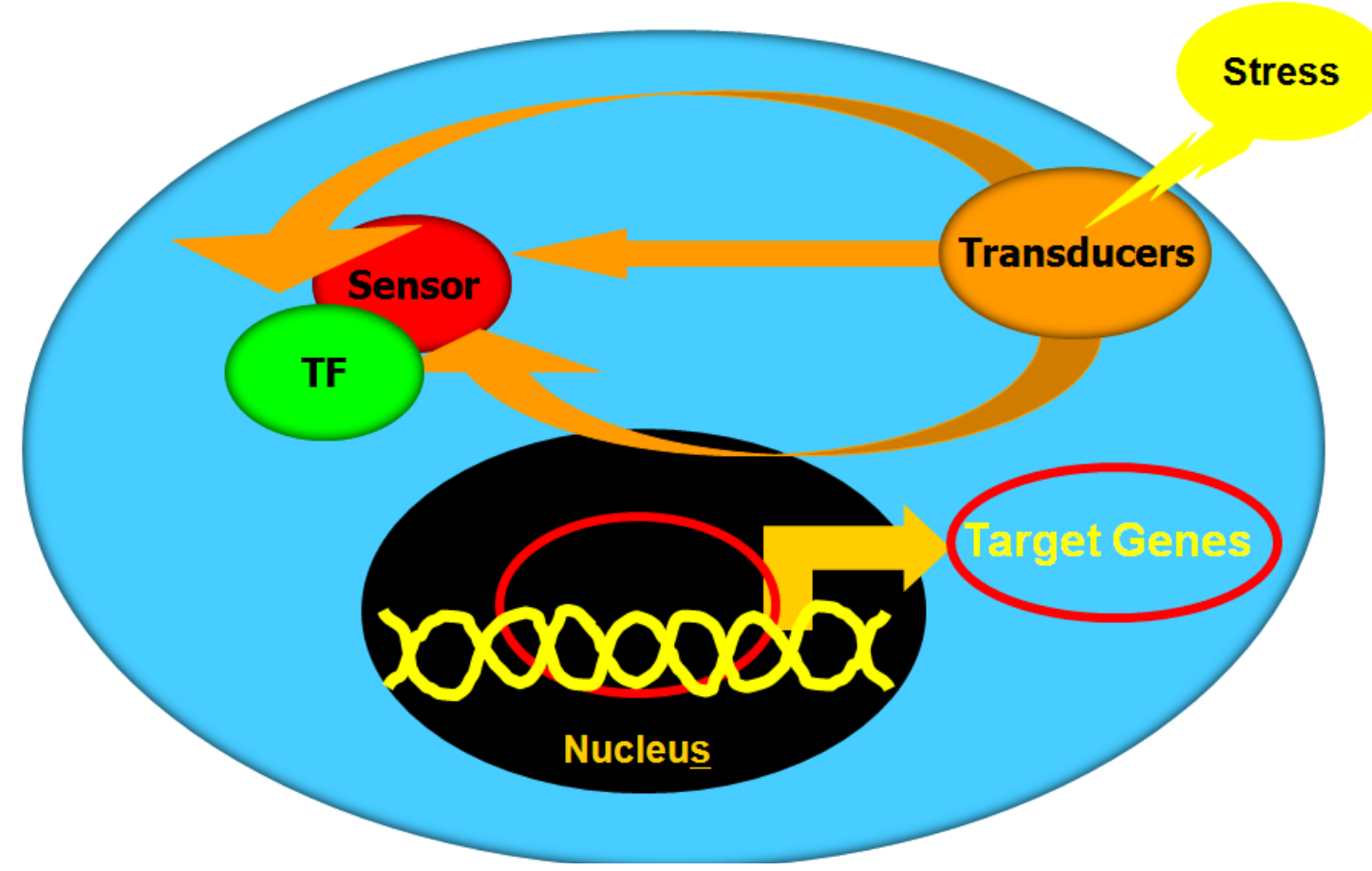

Fig 2. Schematic Model of the Cellular Mechanisms activated by Stress

of Human Enhancement. This is one of the reasons that prompted some experts to establish a non-profit medical society for "scientific research, bioethical analysis and the enhancement of human health". Due to the increasingly long-lived population, the aim will be also to broaden the knowledge in the field of integrated and natural therapies in the aim of preventing aging and most of the pain pathologies of the elderly.

\section{REFERENCES}

1. Watson J. Type 2 diabetes as a redox disease. The Lancet. 2014;9919(383):841-843.

2. Mason HS, North JC, Vanneste M. Microsomal mixed-function oxidations: the metabolism of xenobiotics. Fed Proc. 1965;24(5):1172-1180.

3. Steven OS, Chun-Yang F, Ram R. Cellular Stress Response Pathway System as a Sentinel Ensemble in Toxicological Screening. Toxicol Sci. 2009;111(2):202-225.

4. Pecorelli A, Bocci V, Acquaviva A, Belmonte G, Gardi C, Virgili F, et al. NRF2 activation is involved in ozonated human serum upregulation of HO-1 in endothelial cells. Toxicol Appl Pharmacol. 2013 Feb;267(1): 30-40. doi: 10.1016/j.taap.2012.12.001.

5. Re L, Martinez-Sanchez G, Bordicchia M, Malcangi G, Pocognoli A, Morales-Segura $\mathrm{MA}$, et al. Is ozone pre-conditioning effect linked to Nrf2/EpRE activation pathway in vivo? A preliminary result. Eur J Pharmacol. 2014;742:158-62. doi: 10.1016/j.ejphar. 2014.08.029. 